\title{
Critical behavior of ion mobilities near the superfluid transition*
}

\author{
D. Goodstein \\ Laboratori Nazionali del Comitato Nazionale per l'Energia Nucleare, Frascati, Roma, Italy \\ and California Institute of Technology, Pasadena, California $91109 \dagger$
}

A. Savoia and F. Scaramuzzi

Laboratori Nazionali del Comitato Nazionale per l'Energia Nucleare, Frascati, Roma, Italy

(Received 9 January 1974)

\begin{abstract}
Measurements are reported of the mobilities of helium ions in superfluid helium very close to the $\lambda$ transition. The mobilities of both positive and negative ions appear to be singular in the sense of having infinite slope at the transition. The measurements are made by a new differential technique, based on the space-charge-limited method, which makes possible a sensitivity to changes in mobility of the order of parts in $10^{4}$. This allows mobility measurements into the range $|\epsilon|<10^{-4}$, where $\epsilon=\left(T / T_{\lambda}\right)-1$. Most of the reported measurements are taken along isotherms which cross the $\lambda$ line at elevated pressures, although a series at saturated vapor pressure is also reported. Means of transforming the data between various thermodynamic paths are discussed. Uncertainty as to which thermodynamic path should be considered fundamental together with experimental problems in the technique at this stage in its development make quantitative assessment of the singularity difficult. However, if we assume a limiting form $\left(\mu-\mu_{\lambda}\right) / \mu_{\lambda} \sim(-\epsilon)^{\rho^{\prime}}$, where $\mu$ is the mobility and $\mu_{\lambda}$ its value at the transition, we find for the exponent $\rho^{\prime}=0.94 \pm 0.02$.
\end{abstract}

\section{INTRODUCTION}

Over the past 15 years, the study of the behavior of ions in liquid helium has proved a particularly fruitful source of information about the microscopic nature of the superfluid state. More recently, much attention in the study of helium has been directed towards the $\lambda$ transition, the phase transition between normal and superfluid helium. In this paper, we wish to present the results of measurements of ion mobilities very close to the $\lambda$ transition as it is approached from the super fluid side.

The $\lambda$ transition is now understood to be representative of a class of phenomena called critical phenomena, which are phase transitions without latent heat. These transitions are characterized, in general, by an order parameter whose average value tends to zero as the transition is approached from one side. In helium, the order parameter is usually taken to be the condensate fraction, a quantity which cannot be measured directly, but is thought to be related to the superfluid fraction $\rho_{s} / \rho$. On either side of these phase transitions, but very close to them, fluctuations in the order parameter tend to become correlated over long distances. The measurable thermodynamic properties of the substance involved become dominated by these long-range fluctuations, whose correlation length grows to infinity as the transition is approached from either side. Predictions concerning the strengths of singularities in measurable properties which are based on these ideas are called scaling laws. ${ }^{1}$

When viewed in these terms, the phase transitions are seen to be essentially macroscopic phenomena, with a characteristic scale, or length, which tends to infinity. Ions, on the other hand, are essentially microscopic probes, with a scale of their own-the ion diameter - on the order of $10 \AA$. Thus, it is not evident a priori that ions will be useful for examining the properties of helium at the phase transition. The data reported in this paper, however, indicate that ion mobilities are singular at the transition, passing through it with infinite slope.

There is precedent provided by singular behavior in analogous measurements near other phase transitions. Electrical resistivities have been found to have singular behavior at the critical points of a number of antiferromagnetic materials, ${ }^{2}$ at the ferromagnetic Curie point in nickel, ${ }^{3}$ at the order-disorder transition in $\beta$ brass, ${ }^{4}$ and at the liquid-gas critical point in the electrolytic solution isobutyric acid-water. ${ }^{5}$ In the magnetic cases, as well as in $\beta$ brass, the charge carriers are conduction electrons, which should be thought of as microscopic objects limited in size by their mean free paths. In the electrolytic solution the charge carriers are ions of both signs.

The most successful theory of this type of phenomenon is an argument in the spirit of the scaling laws presented by Fisher and Langer. ${ }^{6}$ In the ferromagnetic case, for example, they would expect electrons to be scattered in a way that is proportional to the magnitude of the short-range 
part of the spin-spin correlation function. Since the magnetic energy is proportional to the same quantity, they predict that the temperature derivative of the resistivity will be proportional to the temperature derivative of the energy, i.e., to the specific heat. Subsequent experiments have confirmed this prediction in the magnetic materials $\mathrm{s}^{2,3}$ and in $\beta$ brass, ${ }^{4}$ but not in the case of the electrolytic solution. ${ }^{5}$ In general, the temperature derivative of the resistivity appears to diverge at the transition, as does the heat capacity. In the electrolytic solution, however, the divergence in the resistivity is considerably stronger than in the heat capacity.

In the present work, we find that the temperature derivative of the ion mobility diverges (stated in terms of resistivity, the divergence would be the same), but the data are not in a form that makes them easy to compare with the heat capacity. There is, however, no evident reason why the FisherLanger prediction ought to apply to helium and, in fact, we know of no existing applicable theory whatever.

The mobilities of ions as the $\lambda$ transition is crossed were first measured by Grimsrud and Scaramuzzi, ${ }^{7}$ recently by Sitton and Moss, ${ }^{8}$ and by Ahlers and Gamota. ${ }^{9}$ These measurements, however, have not been close enough to the transition to be useful in testing scaling type ideas. Scaling arguments always predict the limiting behavior as the transition is approached. The figure of merit is the distance in temperature from the transition, expressed in a nondimensional way

$$
\epsilon=\left(T-T_{\lambda}\right) / T_{\lambda},
$$

where $T_{\lambda}$ is the transition temperature. There is, of course, no absolute criterion for how small $\epsilon$ must be before scaling ideas become applicable, but we can appeal for guidance to experience gained from the measurement of other parameters, such as the heat capacity ${ }^{10}$ and the superfluid fraction. ${ }^{11,12}$ Generally speaking, helium seems to take on its limiting behavior for $|\epsilon|$ about $10^{-3}$ or less. The mobility measurements cited above do not go below $|\epsilon| \approx 5 \times 10^{-3}$. The present results extend this range, on the superfluid side of the transition, two orders of magnitude, into the $10^{-5}$ range. At $-\epsilon \approx 10^{-3}$, the mobility of positive ions is about $2 \%$ higher than its value at $\epsilon=0$. Conventional techniques for measuring ion mobilities cannot resolve changes much smaller than this, and that is why there have been no truly critical measurements of ion mobilities before the present work. In this paper we present a differential technique capable of detecting changes in mobility on the order of parts in $10^{4}$. This technique has made the critical measurements possible.
In Sec. II we describe the conventional parts of the apparatus used in our measurements and Sec. III is devoted to a detailed description of the differential technique and its present limitations. The results are presented and analyzed in Sec. IV, and our conclusions are presented in Sec. V.

\section{GENERAL EXPERIMENTAL FEATURES}

The main body of the data presented here consists of mobility measurements along isotherms in the $P-T$ plane going very close to the $\lambda$ trans-
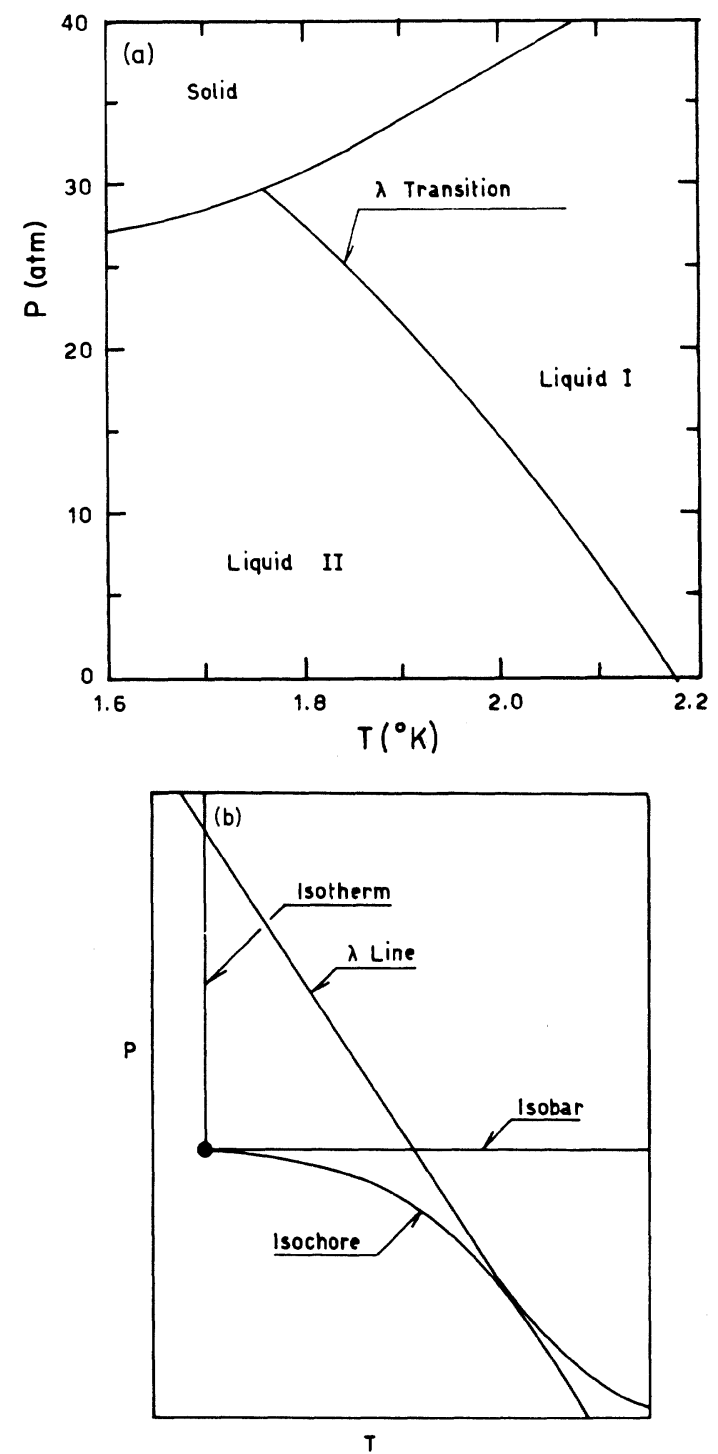

FIG. 1. $P-T$ phase diagram for $\mathrm{He}^{4}$ in the vicinity of the $\lambda$ line: (a) shows the entire $\lambda$ line, (b) is a detail of (a) showing three different possible paths to the transition from a point in the superfluid region: an isotherm, an isobar, and an isochore. Most of the data presented in this paper are taken along isotherms. 
ition from the superfluid side (see Fig. 1). The mobility changes by only a few per cent over the entire range of each isotherm. Since conventional techniques of ion mobility measurement do not resolve changes much smaller than this, a new, more sensitive differential method had to be devised. In essence, the new method consists of using a vibrating reed electrometer as a null meter, by subtracting a fixed reference current from the current gathered in an experimental cell. A detailed discussion of the method is reserved for Sec. III, after we have first described the cells themselves, and all of the conventional aspects of the apparatus.

The apparatus used is shown in Fig. 2. It consists of a double-cell system contained in a main body $A$, made out of oxygen-free high-conductivity copper, which assures a good thermal contact between the two cells and the external thermoregulated helium bath. Stainless-steel flanges B, fastened to $\mathrm{A}$ by indium $\mathrm{O}-\mathrm{rings}$, allow gas and electrical connections to the two cells. Each of the two cells contains a diode $\mathrm{C}$, which will be described below and a carbon-resistor thermometer $D$. The bath is thermoregulated with the help of a germanium thermometer $E$ and a heater F. Two capillary stainless-steel tubes $G$ in thermal contact with the external helium bath are used to control and measure the pressure in each cell. Each diode (see Fig. 3) consists of an emitter $\mathrm{A}$, which is a silver disk coated with $\mathrm{Po}^{210}$, and two silvered brass electrodes facing it, the col-

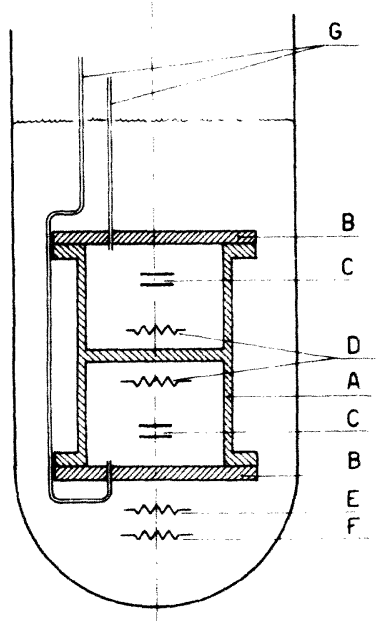

FIG. 2. Schematic diagram of the apparatus: $A-$ main body, oxygen-free high-conductivity copper, B-stainless-steel flanges, C-diodes (for detail, see Fig. 3), D-carbon-resistor thermometers, E-germanium thermometer, F-thermoregulator heater. lector B, and a guard ring C: The diameter of the collector is smaller than that of the emitter in order to avoid edge effects in measuring cur rents. Perspex (D) is used as the insulator in mounting the diode. The two cells are identical: One of them was used as a measuring cell for all experimental data presented here, and the second one was used as a reference cell in some of the runs (see Sec. III). In each cell, the collector (B) is connected to the high impedance input of a vibrating reed electrometer, the guard ring $(C)$ is grounded, and the emitter (A) is connected to a dc voltage generator in order to obtain uniform electric field of the desired sign between the electrodes.

The external liquid helium bath was thermoregulated with a traditional germanium thermometer-heater system, using a lock-in amplifier as the null detector of the bridge. The temperature was kept constant to better than $2 \times 10^{-5} \mathrm{~K}$.

The pressure was monitored by a Bourdon pressure gauge, ${ }^{13}$ with which it was possible to detect the pressure and keep it constant within $\delta P / P$ $\approx 2 \times 10^{-5}$.

The value of the pressure at the $\lambda$ transition was determined with the help of the carbon resistor located within the cell (D, Fig. 2). The procedure was the following: With the bath thermoregulated, a point at a pressure $P>P_{\lambda}$ was reached; then pressure was released in order to cross the $\lambda$ line isothermally. On the same strip-chart recorder the zero of the thermometer bridge and the output of the pressure gauge were displayed: When crossing the $\lambda$ line a jump in temperature (decrease) was produced, cue to the abrupt change in thermal conductivity of the liquid, and $P_{\lambda}$ was thus measured. To begin, rapid passages were performed in order to identify roughly the value of $P_{\lambda}$; these were characterized by big temperature

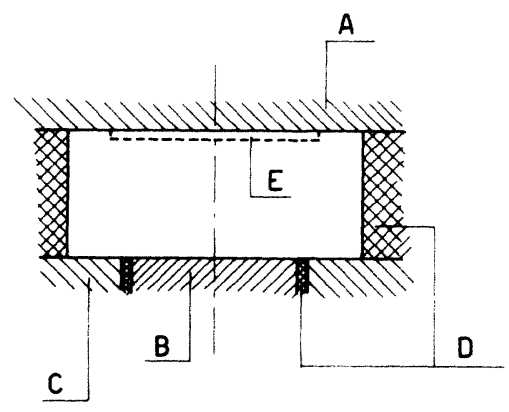

FIG. 3. Schematic diagram of a diode: A-silver disk coated with $\mathrm{Po}^{210}, \mathrm{~B}$-collector, C-guard ring, $\mathrm{D}$-perspex insulation, E-ionized layer (in liquid $\mathrm{He}^{4}$ the thickness of this layer is about $0.2 \mathrm{~mm}$ ); the outer surface of this layer towards the collector is the ion source. 
changes, as well as lack of precision in the value of $P_{\lambda}$ due to pressure gradients in the tubes connecting the cell with the pressure gauge. Then successively slower passages through the $\lambda$ line were performed in order to increase the accuracy of the measurement of $P_{\lambda}$, until the measured value was independent of the passage velocity (some $0.5 \mathrm{Torr} / \mathrm{min}$ at pressures of 10-30 atm). In this way, the value of $P_{\lambda}$ was known with an accuracy of $\approx 1$ Torr (corresponding to $\delta P / P$ $\approx 5 \times 10^{-5}$ ). Differences between values of $P_{\lambda}$ measured at different times in the same run (some $3 \mathrm{~h}$ apart) were typically a factor two larger, giving rise to the errors in $-\epsilon$ shown in Fig. 12 (s€e Sec. IV).

One of the present limits of these measurements is connected with the presence of a rather intense radioactive source in the cell. The source must be intense in order to create the condition of full space charge limitation (see Sec. III), but at the same time it is a rather intense heat source. Most of the data presented here are obtained with a $\mathrm{Po}^{210}$ source of about $200 \mu \mathrm{Ci}$ distributed over a surface area of about $3 \mathrm{~cm}^{2}$. This corresponds to a heat source of $6.5 \times 10^{-6} \mathrm{~W}$. While this heat leak does not affect measurements in the superfluid region (rather than a temperature gradient, counterflow velocities less then $10^{-5} \mathrm{~cm} / \mathrm{sec}$ are set up), it renders practically impossible measurements in the normal region. It can be roughly estimated that such a heat source would produce in He I a temperature difference of the order of $10^{-3} \mathrm{~K}$ across the experimental space, which is almost the entire temperature range in which the measurements in the superfluid region presented here lie. Work is in progress on the task of extending the measurements into the normal region.

\section{MEASUREMENT OF MOBILITY IN THE ASYMPTOTIC REGION}

The technique used for the measurement of the mobility is based on the "full-space-charge" (FSC) method $^{14}$ which we describe briefly here. In a diode with a simple geometry (see Fig. 3) an intense ion source is created and a voltage of the desired sign is applied between the electrodes. We use parallel plane electrodes, one of which is coated with $\mathrm{Po}^{210}$, an $\alpha$ emitter which creates a thin, well-defined, intensely ionized layer (E) within the liquid. The outer surface of this plasma serves as the ion source. If the FSC condition holds, the ion current moving from the ion source to the collector completely shields the source, so that the electric field there is zero. Then Poisson's equation is easily integrated to give $\mathrm{e}^{15}$

$$
i=\alpha \mu V^{2}, \quad \alpha=9.95 \times 10^{-14} S / l^{3},
$$

where $i$ is the current collected (in A), $S$ is the surface area of the collector (in $\mathrm{cm}^{2}$ ), $l$ is the distance between the collector and the ion source (in $\mathrm{cm}$ ), $V$ is the applied voltage (in $\mathrm{V}$ ), and $\mu$ is the mobility (in $\mathrm{cm}^{2} / \mathrm{V} \mathrm{sec}$ ). A plot of $i$ vs $V^{2}$ is a straight line whose slope gives $\mu$, provided that $\alpha$ is well known. Conversely, the observation that such a plot is a straight line gives experimental evidence that the FSC condition is satisfied. Figure 4 gives examples of such plots, obtained with the apparatus described in this paper, for different mobilities. Used in this way the method permits the measurement of mobility (in the liquid-helium region) with an absolute accuracy of about $10 \%$ and a sensitivity to changes of the order of $1 \%$. An improvement of the sensitivity is necessary in order to obtain meaningful information in the asymptotic region: For $\epsilon=\left(T-T_{\lambda}\right) / T_{\lambda}$ $=10^{-3}$, the mobility differs only about $2 \%$ from its value at the $\lambda$ transition.

We have devised a method for this experiment which has two important advantages over conventional procedures. First, it increases the sensitivity by a factor $\approx 100$, and second, it permits the deduction of the value of $\mu$ with a single voltage measurement and thus shortens the measuring times. It consists in performing the measurements at constant current $i_{0}$ and in using a vibrating reed electrometer as a zero meter, by sending into it a signal which nulls the current $i_{0}$. The sensitivity of the electrometer can then be increased by a factor $\approx 100$, being finally limited by noise in the current. With this method,

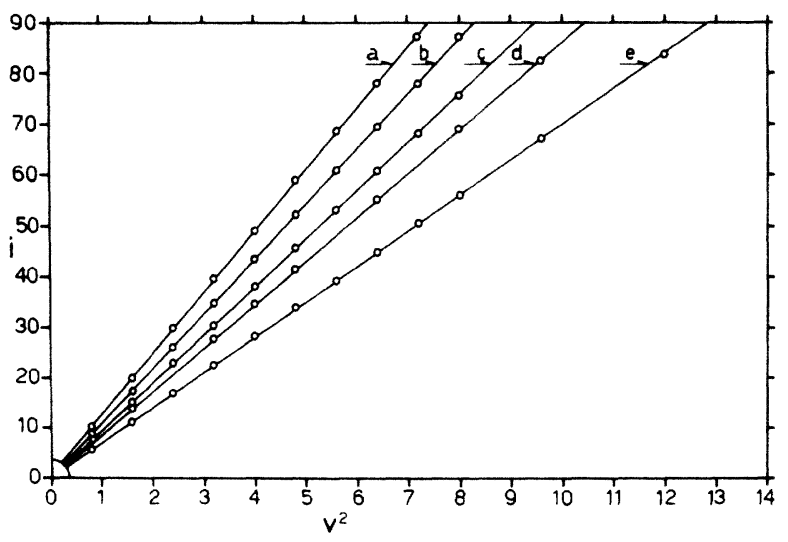

FIG. 4. Plots of $i$ vs $V^{2}$ in conditions of full-spacecharge limitation, for different temperatures at standard vapor pressure. The value of the mobility is proportional to the slope of the experimental straight lines. In the graph, $i$ is in arbitrary units, $V^{2}$ is in $\mathrm{V}^{2}$ (run 34). a-T $=2.003 \mathrm{~K}, \mu=8.697 \times 10^{-2} \mathrm{~cm}^{2} / \mathrm{V} \mathrm{sec}$; $\mathrm{b}-T=2.048 \mathrm{~K}, \mu=7.797 \times 10^{-2} \mathrm{~cm}^{2} / \mathrm{V} \mathrm{sec} ; \mathrm{c}-T=2.095 \mathrm{~K}$, $\mu=6.827 \times 10^{-2} \mathrm{~cm}^{2} / \mathrm{V} \mathrm{sec} ; \mathrm{d}-T=2.124 \mathrm{~K}, \mu=6.207$ $\times 10^{-2} \mathrm{~cm}^{2} /$ V sec; $\mathrm{e}-T=2.172 \mathrm{~K}, \mu=5.049 \times 10^{-2} \mathrm{~cm}^{2} / \mathrm{V} \mathrm{sec}$. 
what is measured when the mobility changes, is the new value of the applied voltage that makes the current equal to $i_{0}$, so that the electrometer once again reads zero. The mobility values will then be proportional to $1 / V^{2}$, the proportionality factor being $i_{0} / \alpha$. The data may be quoted in a way that is independent of the proportionality factor by choosing a reference point in the $P-T$ plane, where the mobility is, say $\mu_{1}$, giving a voltage $V_{1}$. Then, at any other point nearby,

$$
\left(\mu-\mu_{1}\right) / \mu_{1}=\left(V_{1} / V\right)^{2}-1 \text {. }
$$

In practice, we choose for our reference point the mobility at the $\lambda$ transition $\mu_{\lambda}$ so that the quantity we measure is

$$
\Delta \mu / \mu=\left(\mu-\mu_{\lambda}\right) / \mu_{\lambda} \text {. }
$$

As we shall see in Sec. IV it is also necessary to specify where on the $\lambda$ line $\mu_{\lambda}$ is taken, but that refinement does not affect the present discussion. Three different techniques were used in different runs in order to null the current in the electrometer. The first method (A) consists in having a double cell in the experimental setup, with identical diodes in the two cells (see Fig. 2). The cells are in the same liquid-helium bath and thus at the same temperature, while the pressure can be changed independently in each of them. A reference point in the $P-T$ plane is chosen and one of the two cells is kept fixed at that point, at constant temperature and pressure ( $r \in f e r e n c e$ cell), while the pressure is changed isothermally in the other one (measuring cell). The two collector electrodes are connected to a "Master and Slave" electrometer system ${ }^{16}$ which is then zeroed at the chosen reference point. Any change in mobility, due to pressure changes in the measuring cell, produces a change in the balance current that can be nulled by changing the voltage applied to the measuring cell. The second method (B) is simply to replace the reference cell with a constant current generator. The third method (C) permits the use of a single electrometer: The current at the reference point is nulled by sending an equal signal of opposite sign in the low-impedance side of the electrometer circuit (by adding an extra emf to the "zero adjust" of the electrometer).

A few figures will help to give an idea of the limits of this technique. With a collector area of $\approx 1.5 \mathrm{~cm}^{2}$, a typical value of $i_{0}$ for which the FSC condition held was $2.5 \times 10^{-12} \mathrm{~A}$; each one of the three nulling methods could detect a change of $\approx 2 \times 10^{-16} \mathrm{~A}$, corresponding in most cases to $\delta V \approx 0.5 \mathrm{mV}$ out of an applied voltage $V \approx 10 \mathrm{~V}$. This corresponds to a change in mobility $\Delta \mu / \mu \approx 10^{-4}$. This limit was consistent with the limits in pressure and temperature control

$$
\Delta P / P \approx \Delta T / T \approx 10^{-5} .
$$

Most of the measurements were taken at constant temperature changing the pressure in a series of stationary values. At each point the value of the voltage $V$ which nulled the current in the electrometer was measured. In order to obtain $V_{\lambda}$ in Eq. (2) above, a linear plot of $V$ versus $P$ was constructed, and a linear extrapolation was made to $P_{\lambda}$ (identified as explained in Sec. II). A typical example is shown in Fig. 5. This procedure assumes that the rate with which $V$ changes as a function of $P$ does not change rapidly at $P_{\lambda}$. In analyzing the data in Sec. IV we come to the opposite conclusion, that the mobility has infinite slope at the $\lambda$ line. Clearly, our extrapolation procedure cannot be responsible for that conclusion, since it has the opposite effect on the data. In any case, the extrapolation is short and introduces very little uncertainty. At most, the point or two closest to the $\lambda$ transition in each run may be systematically biased a bit to low values of $\Delta \mu / \mu$.

Since we are using the FSC method with a sensitivity orders of magnitude greater than ever attempted before, errors that are usually negligible now become important. We do not yet fully understand all of the difficulties that have come to light, but we are able to discuss them in terms of the effects they are observed to have upon our results. For the sake of clarity, we distinguish three classes of errors: those that affect the absolute value of the mobility (common to all FSC mea-

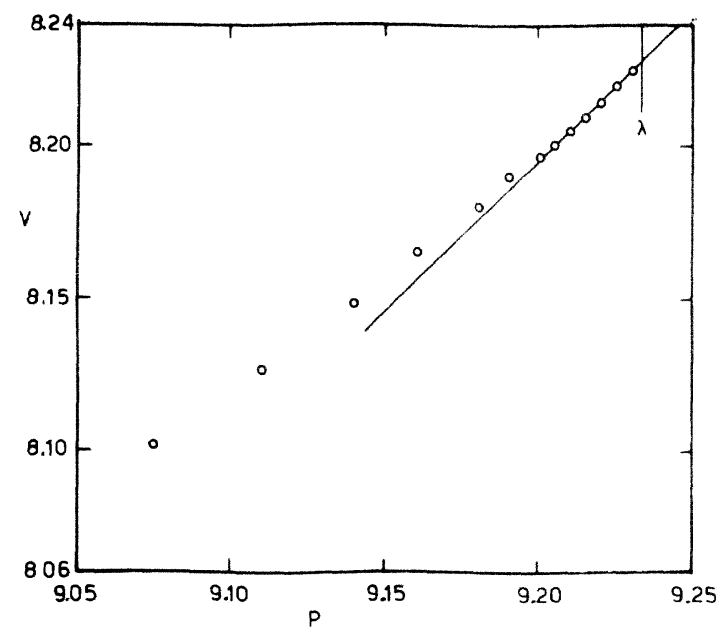

FIG. 5. Determination of $V_{\lambda}$ as a linear extrapolation of the nulling voltage $V$ as a function of the pressure $P$ at the $\lambda$ transition ( $r$ un 28). The vertical line indicates the value of $P$ (in arbitrary units) at which the transition occurs along the isotherm ( $T=2.006 \mathrm{~K}$ in this case). The value of $V_{\lambda}$ is given by the crossing of the two lines. 
surements), those that limit the sensitivity to changes in mobility (and are, therefore, most important when small changes are being measured, very close to the transition), and those that introduce errors into all relative measurements, even when the changes measured are rather large.

A. Systematic errors which affect the absolute value of the mobility

These are due to errors in the measurements of $i, V, S$, and $l$, of which the most important are those connected with $i$ and $l$. The errors in $i$ are due to the low value of the currents usually measured $\left(10^{-10}-10^{-15} \mathrm{~A}\right)$ : a vibrating reed electrometer is used and the value of its input resistance is not normally known to better than $\approx 5 \%$. The errors in the measurement of $l$ are important since it appears to the third power in Eq. (1). Furthermore, $l$ is actually the distance between the ion source and the collector, i.e., between the surface of the ionized layer and the collector, rather than the distance between the electrodes. The thickness of the ionized layer is given by the range of the $\alpha$ particles and therefore, depends on the density of the medium. This effect was corrected for in computing the absolute value of the mobility at widely separated pressures, but it was negligible within any single run of differential measurements since the pressure then changed by no more than about $1 \%$. The absolute values of $\mu$ reported in Sec. IV have been normalized to agree with the data of Ahlers and Gamota ${ }^{9}$ at the saturated vapor pressure, thus calibrating our geometry and the input resistance of the electrometer. The normalization required a change of about $6 \%$ from our own calculations of these factors, well within the expected uncertainties.

\section{B. Errors that limit the sensitivity of the method}

They are mostly due to limits in current and voltage measurements, and temperature and pressure measurement and control, as well as determination of the $\lambda$ point in each run. They combine to introduce an uncertainty of about $\pm 10^{-5}$ in $|\epsilon|$ and $\pm 10^{-4}$ in $\Delta \mu / \mu$. These limits are consistent, in the sense that all of them must be improved in order to obtain a more precise measurement. As we shall see in Sec. IV, the most important information to be inferred from our results is $\rho^{\prime}$, essentially the slope of a plot of $\log (\Delta \mu / \mu)$ vs $\log (-\epsilon)$. The results quoted for $\rho^{\prime}$ are all obtained from data at values of $-\epsilon$ sufficiently large so that they are not affected by errors of the type we have just quoted.
C. Errors in the differential technique

As we shall see in Sec. IV, although the statistical errors in the determination of $\rho^{\prime}$ in any single run are small, the disagreement between values of $\rho^{\prime}$ in different runs is substantially larger. This type of discrepancy cannot be accounted for by errors of the type discussed above. They must be due to errors that become larger as $\Delta \mu / \mu$ becomes larger, are systematic within any single run, and change from one run to another. These errors presently constitute the principal limitation of the technique, and so merit special consideration.

The source of the difficulty may be seen in a careful analysis of the raw data. It is found that if the pressure and temperature in the cell are kept fixed, the voltage necessary to keep the current fixed, nevertheless, drifts slowly. The observed drift rate changes from day to day, and even at different times within the same day. It is sometimes positive and sometimes negative. Its magnitude is typically $1-10 \mathrm{mV} / \mathrm{h}$, much too small to be detected in conventional FSC measurements, but important at the level of sensitivity at which we are working.

Our normal technique for taking data minimized the effects of this drift. Immediately after finding the $\lambda$ point (see Sec. II), data points were taken starting very close to the transition and moving successively farther away. Thus, the effect of the drift is smallest when the points at small $\Delta \mu / \mu$ are taken, and larger only when the voltage difference we are measuring is also large. The result, obviously, is that the error grows as $\Delta \mu / \mu$ does. We note that, if the error were strictly proportional to $\Delta \mu / \mu$, it would not introduce any error at all into $\rho^{\prime}$. That, of course, does not happen exactly, but we do observe that the discrepancies in $\rho^{\prime}$ are smaller than those in $\Delta \mu / \mu$.

Eventually an improved technique was devised to correct for this kind of drift. A series of points would be taken as described, moving away from the transition. Then a new series would be taken, moving back towards the transition at each of the values of $P$ and $T$ used in the first series. A plot of $V$ vs time is then made, with points at the same $P, T$ connected by lines. Such a plot is shown in Fig. 6. The values of $V$ finally used for each $P$ and $T$ are those at which the lines cross the time $t=423 \mathrm{~min}$. If the drift is linear in time, this has effect of making all of the measurements as if they had been taken at the same instant.

If the drift is linear in time, all of the lines in Fig. 6 should be parallel. This is nearly, but not quite, the case. Thus, even with this correction, 
there are errors due to higher-order time derivatives of the drift. To take a series of data points in this way took more than twice as long as the single-series technique described above, thus increasing the effect of the drift and reducing the efficiency of the corrections. About half the data reported in Sec. IV were corrected in this way (see Fig. 11), but there is no correlation between the results for $\rho^{\prime}$ and the correction technique used.

Possible causes of drift at the levels observed are difficult to track down, but we can make some informed guesses. The principal candidates are drifts in the input resistance to the electrometer and in the distribution of electric fields within the experimental cell.

The very large resistances $\left(10^{10}-10^{12} \Omega\right)$ used at the input of the electrometers surely have substantial temperature coefficients and may be expected to change somewhat as the temperature of the room changes in the course of a working day. The resistor is physically located directly on the upper flange of the experimental dewar, in a box

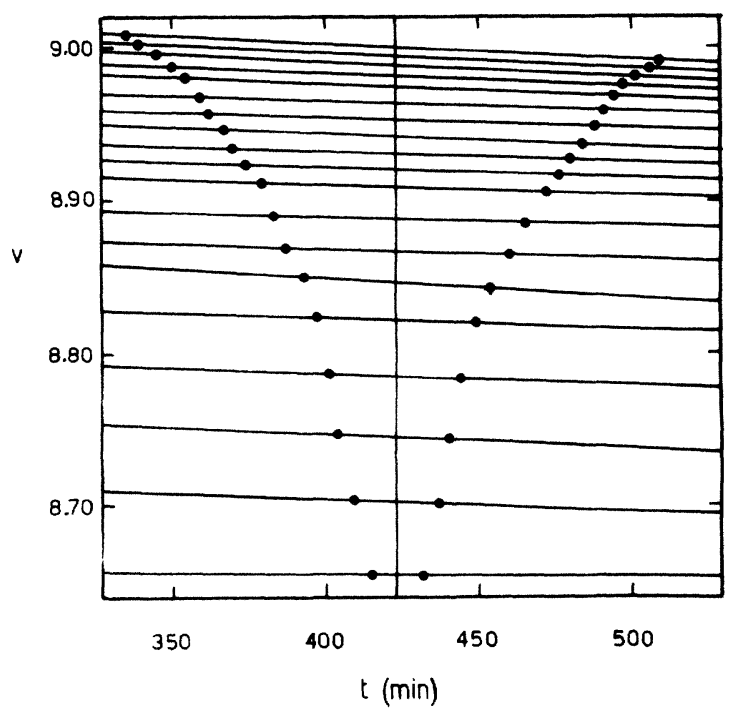

FIG. 6. Plot of the nulling voltage $V$ at fixed temperature and pressure as a function of time $t$. All the points are taken at the same temperature (run 29/2, $T$ $=1.904 \mathrm{~K}$ ). The straight lines connect pairs of points taken at the same pressure at different times. This graph is used as a first approximation correction for the drift of the nulling voltage $V$ as a function of time: If the drift were linear in time, the lines would be parallel, and the correction would be exact. The values of $V$ chosen to calculate $\Delta \mu / \mu$ are those corresponding to a common time, in this case $t=423 \mathrm{~min}$. Data were taken starting soon after a measurement of the $\lambda$ parameters $\left(P_{\lambda}, V_{\lambda}\right)$, by decreasing pressure to the lowest $P$ (corresponding to the lowest $V$ ) and then increasing $P$ up to the $\lambda$ transition again. that is heated above room temperature, reducing its sensitivity to ambient conditions. Moreover, of the three techniques cited above for nulling the current, two of them, $A$ and $B$, made use of two adjacent resistors, so that ambient temperature effects could be expected to cancel; there is no systematic difference noted between the results using those techniques and the results using technique $C$, in which only one resistance is present and the current is nulled on the low impedance side of the input. Nevertheless, we are considering drifts at the level of parts in $10^{5}$, and the input resistance could contribute to them.

The experimental cell inevitably must have insulating materials in it (perspex and PVC), and these slowly become charged during the day, altering the distribution of fields in the working space. Moreover, the $\mathrm{Po}^{210}$ source is undoubtably inhomogeneous, having small insulating areas which change as the source ages. In addition, as the source ages, small quantities of $\mathrm{Po}^{210}$ evaporate, find their way to other parts of the cell, and create local conditions of unpredictable effect. All of these may contribute to small drifts in the current at constant applied field. One sure sign that some such effects were present was that it was found to be impossible to work with positive and negative ions on the same day. Changing the sign of the field gave rise to drifts in the current much stronger than those we are discussing here.

Drifts of this general type are not unfamiliar to workers in the field of ion mobilities. The causes we have discussed were foreseen and some care was taken in the design of the apparatus to minimize them. For example, as mentioned above, two input resistances were used in a Master-Slave arrangement for some of the measurements, and the cell was designed to have relatively little insulating material present. As a result the current was very stable at the level of ordinary FSC measurements (i.e., in our measurements of absolute values of mobilities), but drifts now show up in the much more sensitive differential measurements. Needless to say, this lesson has greatly influenced the design of the next generation of apparatus.

In any case, for the present, the drifts must be taken to be an observed defect in the measuring technique. Our attempts to analyze our data quantitatively, in Sec. IV, are limited in part by the scatter in the results which is effectively introduced by these drifts. However, due to the many different combinations of techniques we have used in making the measurements and analyzing them, and the lack of correlation between the result and the technique, we feel confident that the final errors in measuring $\rho^{\prime}$ are random and not systematic or biased. This point is important in evalu- 
ating the conclusions we reach about the singular behavior of $\Delta \mu / \mu$.

\section{DISCUSSION OF RESULTS}

The overall behavior of the mobility of positive ions in the vicinity of the $\lambda$ transition is shown in Figs. 7-9. These measurements were made by the FSC method, calibrated to agree with the data of Ahlers and Gamota ${ }^{9}$ under saturated vapor pressure, as explained in Sec. III. Figures 7 and 8 , respectively, show measurements along an isobar at $14.19 \mathrm{~atm}$ crossing the $\lambda$ line at $2.007 \mathrm{~K}$, and along an isotherm at $2.001 \mathrm{~K}$ crossing the $\lambda$ line at $14.609 \mathrm{~atm}$. In general, the mobilities descend rapidly as the transition is approached from the superfluid side, cross the transition without any apparent discontinuity, and then change less rapidly in the normal He I region. These observations are consistent with those reported earlier by Grimsrud and Scaramuzzi ${ }^{7}$ at saturated vapor pressure. The slope of the $\lambda$ line at roughly $2 \mathrm{~K}$ and $14 \mathrm{~atm}$ is about $-75 \mathrm{~atm} / \mathrm{deg},{ }^{17}$ so that if the mobility is governed by its distance from the transition, a point in Fig. 85 atm from the transition corresponds to a point in Fig. 7 about 70 mdeg from the transition. Bearing this in mind, we see that the behavior of the mobility is roughly the same whether the transition is approached along an isotherm or an isobar. Figure 9 shows the mobility as a function of temperature and pressure along the $\lambda$ line.

The main body of the data to be presented in this paper consists of differential measurements of the change in mobility as the $\lambda$ line is ap-

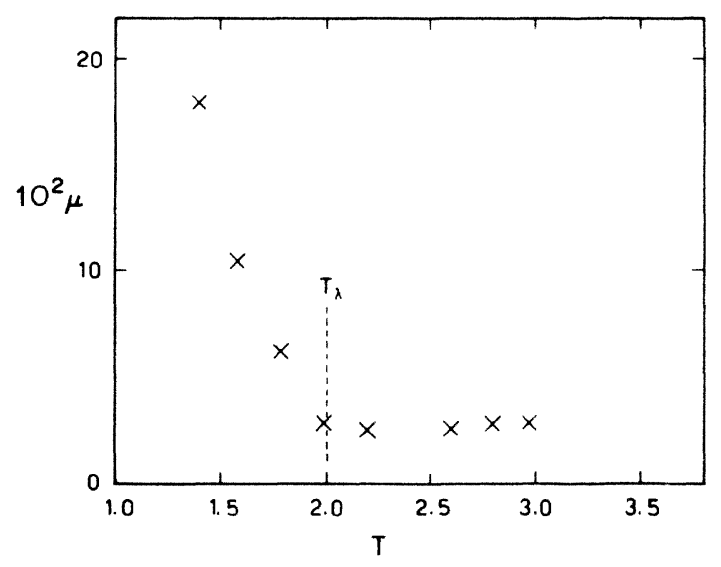

FIG. 7. Mobility of positive ions $\mu$ as a function of the temperature $T$ along an isobar at $P=14.19 \mathrm{~atm}$, crossing the $\lambda$ line at $T=2.007 \mathrm{~K}$. These data are normalized to agree with the data of Ahlers and Gamota (Ref. 9) under saturated vapor pressure (run 33).

$\mu$ is in $\mathrm{cm}^{2} / \mathrm{V} \mathrm{sec}, T$ is in $\mathrm{K}$.

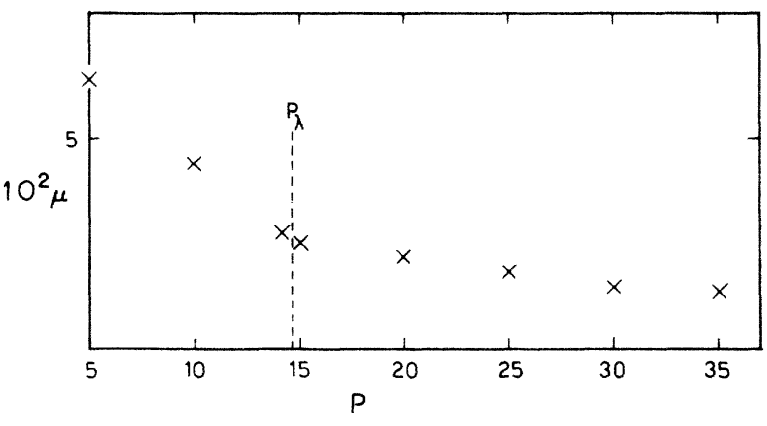

FIG. 8. Mobility of positive ions $\mu$ as a function of pressure $P$ along an isotherm at $\boldsymbol{T}=2.001 \mathrm{~K}$, crossing the $\lambda$ line at $P=14.609 \mathrm{~atm}$. These data are normalized to agree with the data of Ahlers and Gamota (Ref. 9) under saturated vapor pressure (run 33). $\mu$ is in $\mathrm{cm}^{2} /$ $\mathrm{V}$ sec, $P$ is in atm.

proached from below along isotherms, i.e., by changing the pressure at constant temperature. Using a portion of these data (run 28) we show in Fig. 10 a much more detailed isotherm than that shown in Fig. 8. Since the measurements in this case only give changes in $\mu$, we have had to assume that the mobility at the $\lambda$ line on this isotherm $(2.006 \mathrm{~K}$, crossing the $\lambda$ line at 14.241 atm) is exactly $2.85 \times 10^{-2} \mathrm{~cm}^{2} / \mathrm{V}$ sec. The dependence shown in this linear graph does not depend on the choice of $\mu$ and $P$ at which we identify the transition to have occurred. Nevertheless, it is clear from Fig. 10 that the magnitude of the slope of the mobility isotherms increases as the phase transition is approached. The discussion of our data in this section will center around the question of whether the slope becomes infinite in the limit as the transition is approached.

By elementary thermodynamic arguments, it is

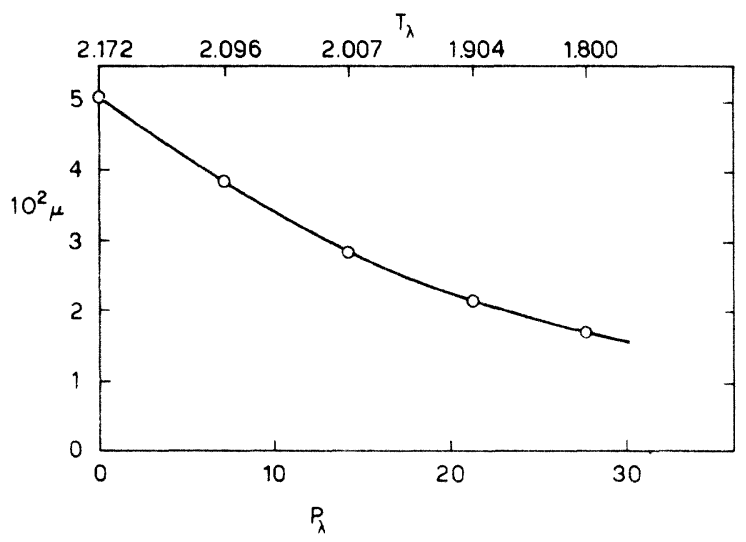

FIG. 9. Mobility of positive ions $\mu$ along the $\lambda$ line as a function of the pressure $P_{\lambda}$ (in the upper scale the corresponding values of $T_{\lambda}$ are indicated). (runs 23, 25,34 .) $\mu$ is in $\mathrm{cm}^{2} / \mathrm{V} \mathrm{sec}, P_{\lambda}$ in atm, $T_{\lambda}$ in $\mathrm{K}$. 
easy to show that the slopes of the mobility as the $\lambda$ line is approached along various paths are connected by the expression

$$
\left(\frac{\partial \mu}{\partial P}\right)_{T}=\frac{1}{\beta_{V}}\left[\left(\frac{\partial \mu}{\partial T}\right)_{V}-\left(\frac{\partial \mu}{\partial T}\right)_{P}\right]
$$

where

$$
\beta_{V}=\left(\frac{\partial P}{\partial T}\right)_{V} \cdot
$$

Here, subscript $V$ signifies an isochore. The magnitude of $\beta_{V}$ increases as the $\lambda$ line is approached from below, ${ }^{18}$ but cannot become greater than the magnitude of the slope of the $\lambda$ line itself $\left(d P_{\lambda} / d T\right) . \beta_{V}$ is therefore finite at the $\lambda$ line. Thus, we see from Eq. (3) above, that any divergence noted in $(\partial \mu / \partial P)_{T}$ is due to the behavior of the mobility along one path or another, and cannot be introduced by singular behavior in the equation of state of helium. In other words, an infinity in the slope of the mobility along an isotherm is sufficient to show that the mobility is singular at

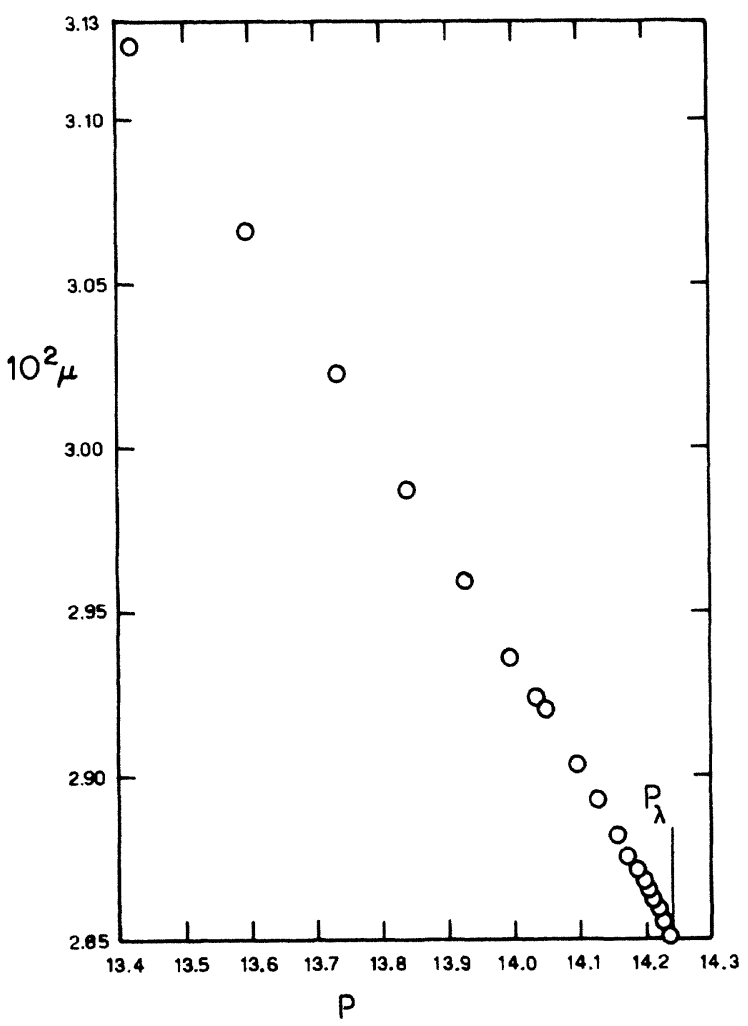

FIG. 10. The mobility of positive ions $\mu$ as a function of the pressure $P$ (in the superfluid region) along an isotherm (run 28, $T=2.006 \mathrm{~K}$ ) in a much more detailed plot than in Fig. 7. $\mu$ is in $\mathrm{cm}^{2} / \mathrm{V} \mathrm{sec}\left(\mu_{\lambda}=2.85 \times 10^{-2} \mathrm{~cm}^{2} /\right.$ V sec), $P$ is in arbitrary units (in these units $P_{\lambda}=14.241$ ). the $\lambda$ transition.

Examples of data taken along isotherms at approximately $1.8,1.9,2.0$, and $2.1 \mathrm{~K}$ are shown in Fig. 11 in the form $\log _{10}\left[(\Delta \mu / \mu)_{T}\right]$ vs $\log _{10}\left[(\Delta P / P)_{T}\right]$, where

$$
(\Delta \mu / \mu)_{T}=\left[\mu-\mu_{\lambda}(T)\right] / \mu_{\lambda}(T)
$$

and

$$
(\Delta P / P)_{T}=\left[P-P_{\lambda}(T)\right] / P_{\lambda}(T),
$$

where $\mu_{\lambda}(T)$ and $P_{\lambda}(T)$ are, respectively, the mobility and pressure on the $\lambda$ line at the temperature of the isotherm. We see that the data plotted in this way give apparently straight lines over approximately three decades in both $(\Delta \mu / \mu)_{T}$ and $(\Delta P / P)_{T}$. If we call the slopes of these lines in the $\log -\log$ plot $\rho^{\prime}$, then the dependence implied by the lines is

$$
(\Delta \mu / \mu)_{T}=A(-\Delta P / P)_{T}^{\rho^{\prime}}
$$

and

$$
\left(\frac{\partial \mu}{\partial P}\right)_{T} \propto\left(-\frac{\Delta P}{P}\right)_{T}^{\rho^{\prime}-1} .
$$

Careful examination shows that all of the lines have slope slightly, but unmistakably less than one. Thus, in the limit as $\Delta P \rightarrow 0^{-},(\partial \mu / \partial P)_{T} \rightarrow \infty$ according to Eq. (5) above. The behavior shown here is consistent with all of the data we have taken, including nine isotherms for positive ions, two for negative ions, and one run for positive ions varying temperature along the saturated

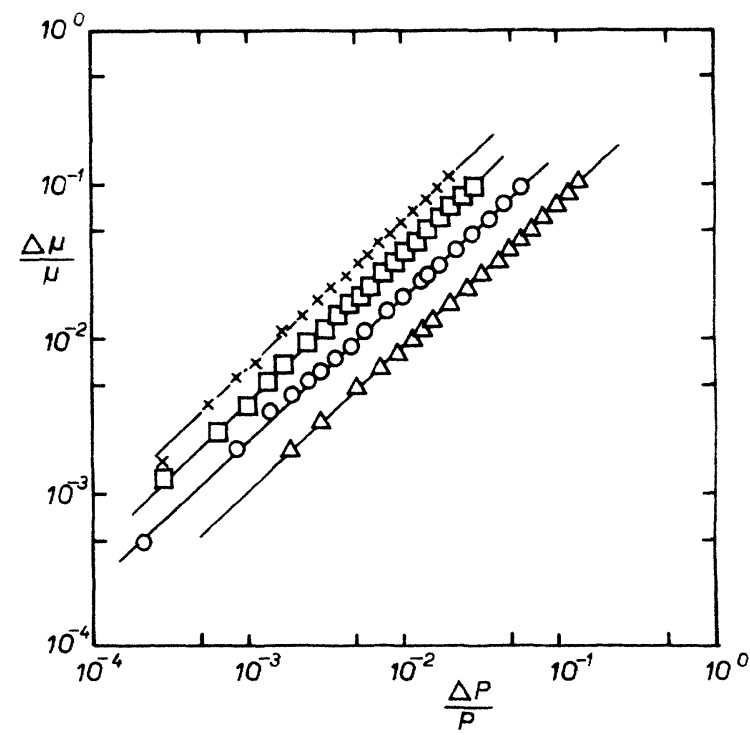

FIG. 11. Plot of $\log _{10}\left[\left(\mu-\mu_{\lambda}\right) / \mu_{\lambda}\right]$ for positive ions as a function of $\log _{10}\left[\left(P-P_{\lambda}\right) / P_{\lambda}\right]$ for different temperatures : $\times, T=1.804 \mathrm{~K}$ (run 29/3); $\square, T=1.904 \mathrm{~K}$ (run 29/ $2) ; \bigcirc, T=2.006 \mathrm{~K}$ (run 28); $\triangle, T=2.094 \mathrm{~K}$ (run 29/1). 
vapor pressure.

As we have already seen, it is convenient to express the results in terms of the distance in temperature from the $\lambda$ transition. This is particularly useful for comparing our results to other measurements of quantities with critical behavior near the phase transition, and for comparison to mobility measurements along the vapor pressure curve. In accordance with the usual notation ${ }^{19}$ for phenomena near the critical phase transition, we define the quantity $\epsilon$,

$$
\epsilon(T, P)=\left[T-T_{\lambda}(P)\right] / T_{\lambda},
$$

where $T_{\lambda}(P)$ is the $\lambda$ temperature at the pressure $P$. In the region close to the $\lambda$ line (where all of our differential data lie), $\epsilon$ is related to $P-P_{\lambda}(T)$ by

$$
\epsilon=-\left(T_{\lambda} \frac{d P_{\lambda}}{d T}\right)^{-1}\left(P-P_{\lambda}\right)
$$

(in the superfluid $\epsilon$ and $P-P_{\lambda}$ are both negative, as is $\left.d P_{\lambda} / d T\right)$. Obviously, a plot of $\log _{10}(\Delta \mu / \mu)_{T}$ vs $\log _{10}(-\epsilon)$ will have the same slope as those shown in Fig. 11. All of our mobility isotherms for positive ions, plotted in this way, are shown in Fig. 12. From this point on, all of our discussion will be in terms of the parameter $\epsilon$, instead of the equivalent but less informative $\Delta P / P$.

We know of no previous examples of measurements of the behavior of ion mobilities for values of $-\epsilon$ less than about $5 \times 10^{-3}$. This is because, as mentioned above, conventional techniques for measuring ion mobilities are insensitive to changes small compared to a few percent, which is the entire change in $\mu$ for smaller values of $-\epsilon$. Thus, from the point of view of critical behavior, the results presented here represent a substantial advance in technique, extending the accessible range of $-\epsilon$ by some two orders of magnitude. For the sake of comparison, the

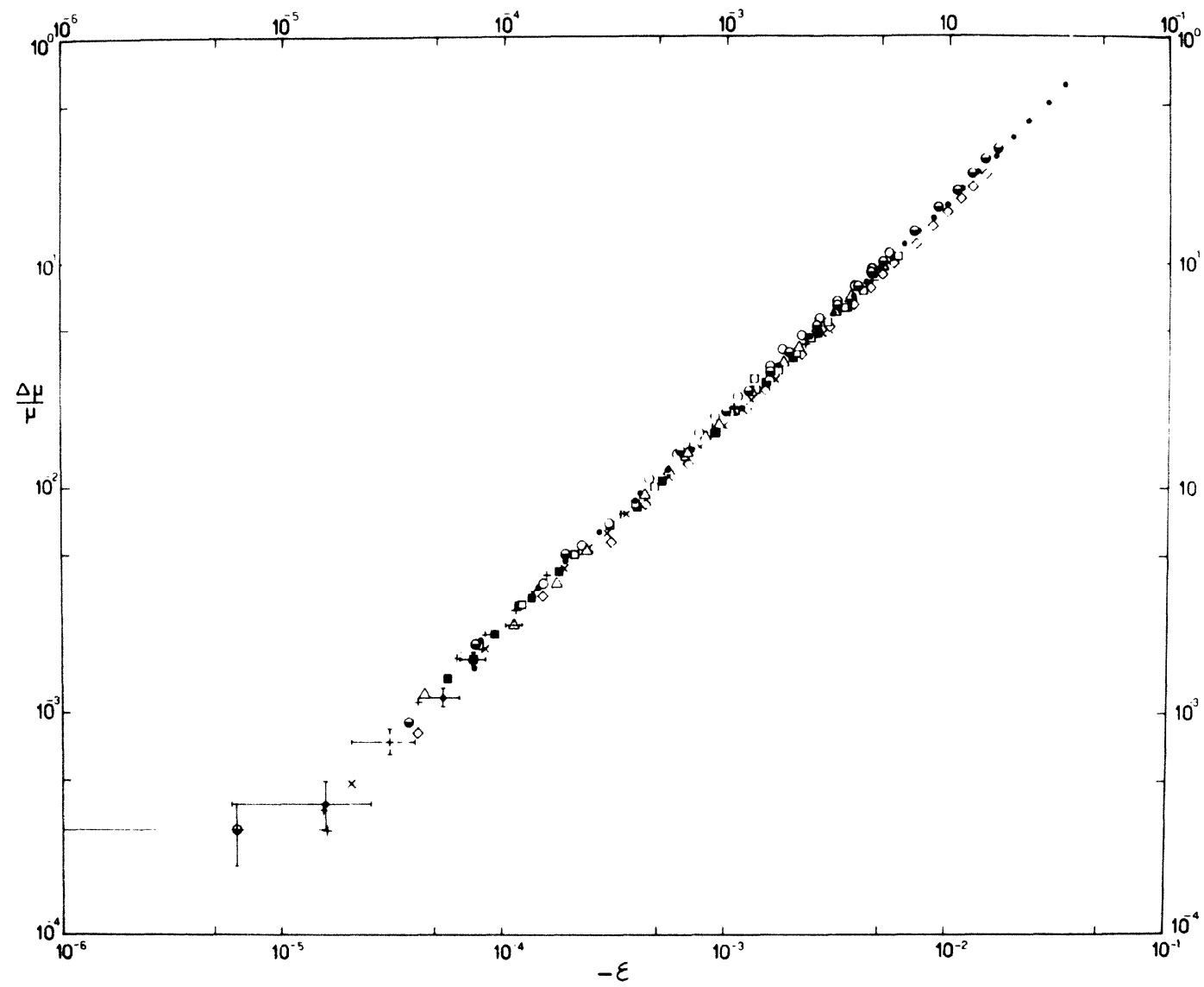

FIG. 12. Plot of $\log _{10}\left[\left(\mu-\mu_{\lambda}\right) / \mu_{\lambda}\right]$ for positive ions as a function of $\log _{10}(-\epsilon)$ [Eqs. (6) and (7)] along isotherms. All of the data for positive ions are shown in this graph, taken at different temperatures, with different nulling techniques (see Sec. III), analyzed in different ways. The errors shown for the points at low $-\epsilon$ are those due to the uncertainty in the determination of the $\lambda$ parameters $P_{\lambda}$ and $V_{\lambda} . O, T=1.804 \mathrm{~K}$ (run 29/3); $\triangle, T=1.904 \mathrm{~K}$ (run $\left.29 / 2\right) ; \square, T=2.094 \mathrm{~K}$ $($ run 29/1); $\times, T=2.006 \mathrm{~K}$ (run 28);,$+ T=2.006 \mathrm{~K}$ (run 27); $\bullet, T=1.800 \mathrm{~K}$ (run 23);,$T=2.098 \mathrm{~K}$ (run 25$) ; \diamond, T=1.999 \mathrm{~K}$ (run 14) ; $\ominus, T=1.804 \mathrm{~K}$ (run 16). 
heat capacity $C_{P}$ reaches its asymptotic behavior only for $-\epsilon \leqslant 10^{-3}$ in superfluid helium. These measurements should thus establish ion mobilities as an additional probe of critical behavior near the superfluid transition.

Since all of the data in Fig. 12 appear to follow a law of the type Eq. (4), let us follow the conventional procedure used in studies of critical phenomena ${ }^{1}$ and formally define a critical exponent $\rho^{\prime}$ for ion mobilities by

$$
\rho^{\prime}=\lim _{-\epsilon \rightarrow 0} \frac{\log _{10}\left[(\Delta \mu / \mu)_{T}\right]}{\log _{10}(-\epsilon)}
$$

(as is also conventional, we reserve the unprimed exponent $\rho$ for later measurements on the normal side of the transition). With this definition we can represent all of the data in Fig. 12 by the approximate equation

$$
(\Delta \mu / \mu)_{T}=a(t)(-\epsilon)^{\rho^{\prime}},
$$

where $a(t) \approx 13$ and does not change very much with $T$ (i.e., along different isotherms), and $\rho^{\prime} \approx 0.94$. We shall return below to a more precise quantitative analysis of these parameters.

Data taken along the vapor pressure curve (which are very nearly equivalent to an isobar, rather than an isotherm) is shown in Fig. 13. Here, we have plotted $(\Delta \mu / \mu)_{\mathrm{SVP}}$ vs $-\epsilon$, where

$$
(\Delta \mu / \mu)_{S V P}=\left(\mu-\mu_{\lambda S V P}\right) / \mu_{\lambda S V P}
$$

and $\mu_{\lambda \text { SVP }}$ is the mobility at the $\lambda$ point at saturated vapor pressure. These data are not directly comparable to those in Fig. 12 since a different thermodynamic path has been followed, but as we shall see below, in the limit as $-\epsilon-0$, this path should give the same exponent $\rho^{\prime}$ as if an isotherm had been followed. As may be seen in Fig. 12, these

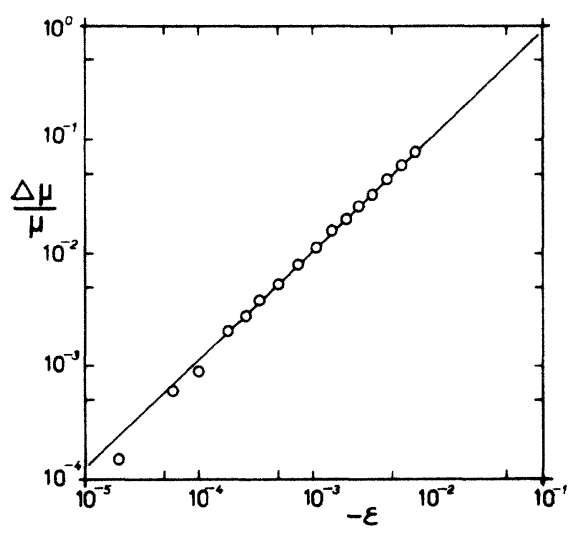

FIG. 13. Plot of $\log _{10}\left[\left(\mu-\mu_{\lambda}\right) / \mu_{\lambda}\right]$ for positive ions as a function of $\log _{10}(-\epsilon)$ [Eq. (6)] along the vapor-pressure curve (run 34). data too follow a straight line in the $\log -\log$ plot. The slope of the line is about 0.96 .

In addition to the data presented for positive ions, we have taken two isotherms for negative ions at $T=2.0 \mathrm{~K}$. These are shown in Fig. 14 . They differ in no important respect from the positive ion data. Since positive and negative ions appear to behave in the same general way, we have devoted our attention up to now to positive ions, and shall restrict the remainder of our analysis to those.

As we have already said, if we fit the data to Eq. (9), with $\rho^{\prime}<1$, then the mobility meets the $\lambda$ line with infinite slope. We wish now to subject the data to analysis designed to test this result. We ask, is it possible that the data represent a mobility which is not singular at the transition?

Let us suppose that the mobility is a regular function of $T$ and $P$ in the vicinity of the phase transition. If there is nothing special about the $\lambda$ line insofar as the mobility is concerned, we should be able to write $\mu(P, T)$ as a Taylor series in $-\epsilon$,

$$
\mu=\mu_{\lambda}\left[1+C_{1}(-\epsilon)+C_{2}(-\epsilon)^{2}+\cdots\right] .
$$

Since $-\epsilon$ is always much less than one in our data, we need not retain terms past $(-\epsilon)^{2}$, but we do retain $(-\epsilon)^{2}$ to see if it is the effect of that term that makes the slope in the log-log plot, Fig. 12, appear to be less than one.

To test for behavior of the type Eq. (10), we have analyzed each isotherm by a linear leastsquares fit to the formula

$$
(-\epsilon)^{-\rho^{\prime}}(\Delta \mu / \mu)_{T}=C_{1}+C_{2}(-\epsilon),
$$

with $C_{1}$ and $C_{2}$ as adjustable parameters. In each attempt, $\rho^{\prime}$ was chosen in advance, and the best values of $C_{1}$ and $C_{2}$ were calculated, along with

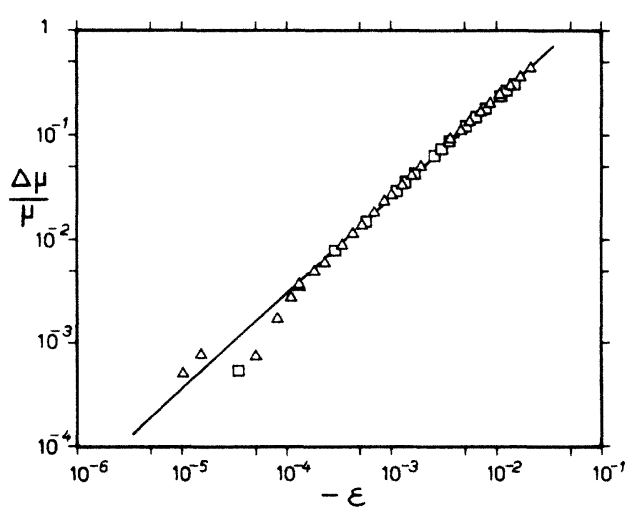

FIG. 14. Plot of $\log _{10}\left[\left(\mu-\mu_{\lambda}\right) / \mu_{\lambda}\right]$ for negative ions as a function of $\log _{10}(-\epsilon)$ [Eqs. (6)(7)] along isotherms : $\square, T=2.000 \mathrm{~K}$ (run 13); $\triangle, T=2.009 \mathrm{~K}$ (run 24). 
the standard error $\sigma$,

$$
\sigma^{2}=\frac{1}{N-m-1} \sum_{i}\left(y-y_{i}\right)^{2},
$$

where for each point, $y(-\epsilon)$ is the right-hand side of Eq. (11) and $y_{i}$ is the left-hand side. $N$ is the number of points and $m$ is the number of parameters. If $(\Delta \mu / \mu)_{T}$ can be written as a Taylor series in $-\epsilon$, we would expect the smallest $\sigma$ to occur for a value of $\rho^{\prime}$ close to one. An example of the results (Run 29/2, isotherm at $T=1.904 \mathrm{~K}$ ) is shown in Table I. The data analyzed in this case lay in the range $1 \times 10^{-4}<-\epsilon<5 \times 10^{-3}$.

A number of striking features show up in Table I. The smallest value of $\sigma$ arises for $\rho^{\prime} \approx 0.96$ and the fit deteriorates seriously if we choose $\rho^{\prime}=1$. Moreover, an unreasonably large value of $C_{2}$ (the second derivative of the mobility in dimensionless units) is required if we are to have $\rho^{\prime}=1$. Finally, $C_{2}$ changes sign at just about the value of $\rho^{\prime}$ that gives the smallest $\sigma$; that is to say, the data reject of their own accord a term of order $(-\epsilon)^{1+\rho}$. These general characteristics held true for all of the isotherms analyzed in this way.

A different technique for testing for Taylor series behavior is shown in Fig. 15. Here, we have plotted as closed circles $(-\epsilon)^{-1}(\Delta \mu / \mu)_{T}$ vs $-\epsilon$, for another isotherm, Run 28 at $T=2.006 \mathrm{~K}$. If Eq. (11) applies, the result should be a straight line with slope $C_{2}$ and intercept $C_{1}$. Obviously, that behavior breaks down at small $-\epsilon$, just where the Taylor series to second order ought to be most accurate. It is possible, however, that the appar-

TABLE I. Test for behavior of the type Eq. (10) (Taylor series). Linear least-squares fit to Eq. (11). $\rho^{\prime}$ is the critical exponent [Eq. (8)], $\sigma$ is the standard error [Eq. (12)], and $C_{1}$ and $C_{2}$ are adjustable parameters. The data are taken from the Run $29 / 2$, isotherm at $T=1.904 \mathrm{~K}$, analyzing only the data which lay in the range $1 \times 10^{-4} \leq-\epsilon \leq 5 \times 10^{-3}$.

\begin{tabular}{cccc}
\hline \hline$\rho^{\prime}$ & $\sigma$ & $C_{1}$ & $C_{2}$ \\
\hline 0.930 & 0.356 & 12.4 & 250 \\
0.935 & 0.348 & 12.9 & 215 \\
0.940 & 0.340 & 13.4 & 176 \\
0.945 & 0.332 & 13.9 & 134 \\
0.950 & 0.326 & 14.5 & 89 \\
0.955 & 0.322 & 15.1 & 41 \\
0.960 & 0.321 & 15.7 & -11 \\
0.965 & 0.323 & 16.4 & -66 \\
0.970 & 0.330 & 17.0 & -126 \\
0.975 & 0.343 & 17.7 & -189 \\
0.980 & 0.362 & 18.4 & -257 \\
0.985 & 0.388 & 19.2 & -329 \\
0.990 & 0.421 & 20.0 & -405 \\
0.995 & 0.462 & 20.8 & -487 \\
1.000 & 0.509 & 21.6 & -574 \\
\hline \hline
\end{tabular}

ent divergence in the slope of the mobility - that is, the refusal of the mobility to follow a Taylor series - is the result of an error in our choice of $\mu_{\lambda}$ and $P_{\lambda}$ (the techniques used to identify these parameters are described in Secs. II and III). To test for that possibility we have reanalyzed the same data, with a new choice for $\mu_{\lambda}$ and $P_{\lambda}$. In choosing these new values, we assumed that all possible sources of error in our procedure combine to change those parameters as far as possible, in such a direction as to make the results to obey a Taylor series. The result of this analysis is shown in the open circles in Fig. 15. Clearly, there is no way the data can be made consistent with Taylor series behavior at the phase transition. All of the isotherms analyzed in this manner gave similar results.

The conclusion we draw from these analyses is that the positive ion mobility is singular, in the sense of having an infinite first derivative, at the $\lambda$ transition. All of the data we have taken is consistent with this conclusion and inconsistent with its reverse. A second, lesser point is that our data do not seem to require higher-order corrections, at least not of order $(-\epsilon)^{1+\rho^{\prime}}$. We shall return to this second point below.

If we may now regard the singular behavior of the mobility as established, we come to the more difficult task of analyzing the singularity quantitatively. The analysis is greatly complicated by the somewhat uncertain nature of the errors in our technique, and, as we shall see below, by the fact that $\rho^{\prime}$ is so close to one.

If the behavior of the mobility along an isotherm $(\Delta \mu / \mu)_{T}$ is known, the behavior along an isobar

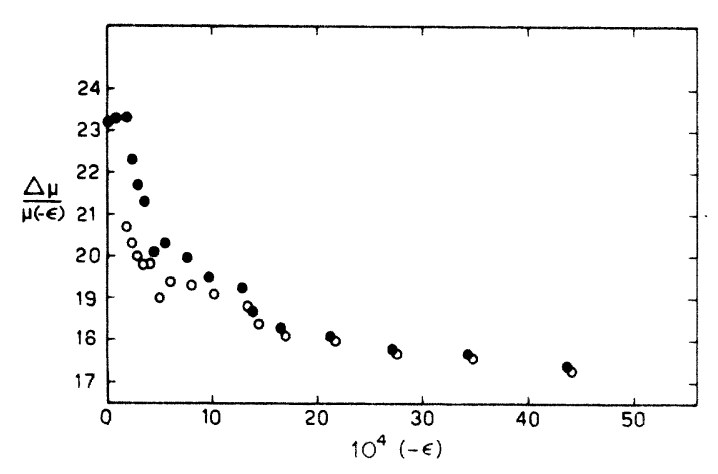

FIG. 15. Plot of $(-\epsilon)^{-1}\left[\left(\mu-\mu_{\lambda}\right) / \mu\right]_{T}$ vs $(-\epsilon)$ (run 28, $T=2.006 \mathrm{~K})$. This plot is a test for Taylor series behavior of $\Delta \mu / \mu$ data as a function of $(-\epsilon)$ [see Eq. (10)]. Closed circles represent average values of $\Delta \mu / \mu$ (the same used in Fig. 12). Open circles are obtained by making, within the limits of errors, an extreme choice for $\mu_{\lambda}$ and $P_{\lambda}$ such as to make the data obey a Taylor series. 
in the same region may be written, to leading or der in $-\epsilon$,

$$
\left(\frac{\Delta \mu}{\mu}\right)_{P}=\left(\frac{\Delta \mu}{\mu}\right)_{T}+\frac{T_{\lambda}}{\mu_{\lambda}} \frac{d \mu_{\lambda}}{d T}(-\epsilon),
$$

where $T_{\lambda}, \mu_{\lambda}$, and $d \mu_{\lambda} / d T$ (the local value of the slope of Fig. 9) may be regarded as constants. Similarly, along an isochore

$$
\left(\frac{\Delta \mu}{\mu}\right)_{V}=\left(\frac{\Delta \mu}{\mu}\right)_{r}+\frac{T_{\lambda}}{\mu_{\lambda}} \frac{d \mu_{\lambda}}{d T} \frac{1}{1-R}(-\epsilon),
$$

where

$$
R=\left(\frac{d P_{\lambda}}{d T}\right)^{-1} T_{\lambda} \epsilon \int_{T}^{T_{\lambda}}\left(\frac{d P}{d T}\right)_{V} d T
$$

with the integral to be carried out along the isochore. The various paths involved may be seen schematically in Fig. 1(b). Thus, in transforming from any one path to any another, a term proportional to $-\epsilon$ is introduced. These terms are nonsingular, and formally vanish in the limit as $-\epsilon$ -0 leaving the behavior

$$
\Delta \mu / \mu \sim(-\epsilon)^{\rho^{\prime}}
$$

along all paths, but, in practice, they render it very difficult to analyze our data for $\rho^{\prime}$. The reason may be seen in Fig. 16, where we have plotted an isotherm at $1.8 \mathrm{~K}$, and also the isobar constructed out of the same data by means of Eq. (13). It is impossible to tell, either from the graph or from a more detailed analysis, which of the two curves (if either) is a pure power law and which has an extra term proportional to $-\epsilon$. However, if we analyze both curves as pure power laws, then the upper curve (the isotherm) appears to have a slightly larger value of $\rho^{\prime}$ (larger by 1 or $2 \%$ ).

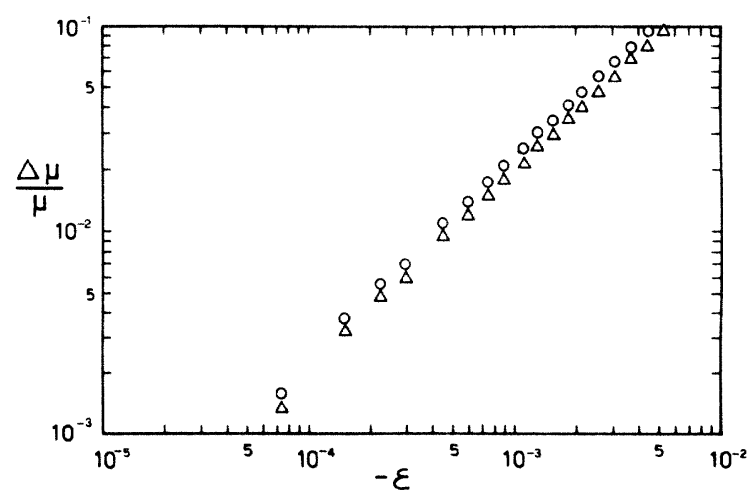

FIG. 16. Plot of $\log _{10}\left[\left(\mu-\mu_{\lambda}\right) / \mu_{\lambda}\right]$ vs $\log _{10}(-\epsilon)$ (run $29 / 3, T=1.804 \mathrm{~K}): O$, the values of $\log _{10}\left[\left(\mu-\mu_{\lambda}\right) / \mu_{\lambda}\right]$ are the original ones, i.e., they are taken at constant temperature; $\triangle$, the same data, transformed into values at constant pressure, following Eq. (13).
It is worth emphasizing at this point that the uncertainty we are now discussing does not cast any doubt on our earlier conclusion that $\rho^{\prime}$ is less than one. This is because, of the paths one might reasonably take to the $\lambda$ line, an isotherm always gives the largest apparent value of $\rho^{\prime}$. From any point in the critical region, $\mu-\mu_{\lambda}$ is larger along an isotherm than along an isobar or an isochore, so that, as in Fig. 16, an isotherm in $\log _{10}(\Delta \mu / \mu)$ vs $\log _{10}(-\epsilon)$ will fall higher than an isobar or an isochore. The curves must come together at small $-\epsilon$, so the isotherms appear to have larger slope. Consequently, the values we deduce from our isotherms represent an upper limit, and it is that upper limit which we have concluded to be less than one.

Nevertheless, we are faced with considerable difficulty in trying to place quantitative values on $\rho^{\prime}$. The well-documented ${ }^{11}$ usual difficulty in extracting critical exponents from experimental data arises in part because of the possible presence of terms of unknown but higher order in $-\epsilon$. In the present case, on the other hand, we feel reasonably confident that terms of genuinely higher order are not present, but we know on thermodynamic grounds that a term of nearly the same order may be present. The term in $-\epsilon$ vanishes so slowly relative to the term in $(-\epsilon)^{\rho^{\prime}}$ (with $\rho^{\prime}$ $\approx 0.94$ ) that we are unable to untangle the two.

To illustrate the point further, we have plotted our data at saturated vapor pressure in the form $\log _{10}\{\Delta \mu /[\mu(-\epsilon)]\}$ vs $\log _{10}(-\epsilon)$ in Fig. 17. In this case, the original data is basically an isobar, and we have used Eq. (13) in reverse to generate the equivalent isotherm. A plot of the kind given here should be a straight line only for a pure power law, and should be curved if there are terms present of different powers, but it is clearly not possible to determine from the data which, if either, of

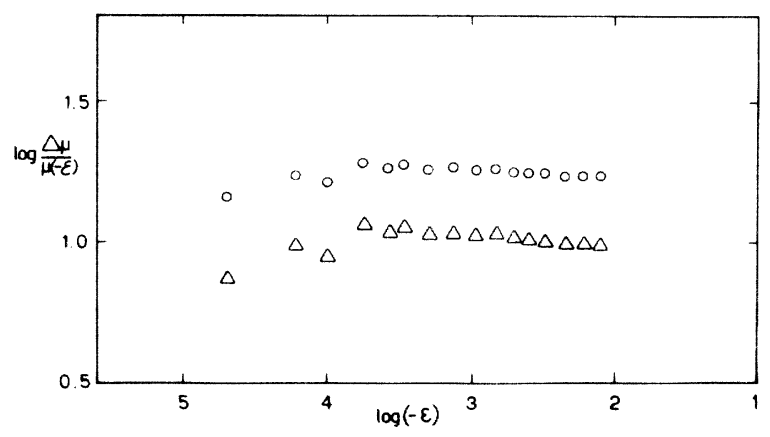

FIG. 17. Plot of $\log _{10}\left\{\left\lfloor\mu-\mu_{\lambda}\right] /\left[\mu_{\lambda}(-\epsilon)\right\rfloor\right\}$ vs $\log _{10}(-\epsilon)$ The data are taken along the vapor pressure curve (run 34), which can be considered basically an isobar: $\triangle$, the original data; $\bigcirc$, the same data, transformed into $\left\{\left(\mu-\mu_{\lambda}\right) /[\mu(-\epsilon)]\right\}_{T}$ following Eq. '13). 
these is the pure power law. Note, incidentally, that both curves have negative slope for $\log _{10}(-\epsilon)>4$, indicating $\rho^{\prime}<1$, but they give slightly different values for the exponent. For the isobar, as mentioned ear lier, the best fit gives $\rho^{\prime}=0.962$, but for the isotherm (which is more comparable with our other data) we get $\rho^{\prime}=0.977$.

In order to analyze the data further, we must make an assumption at this point, and so without further justification, we assume that the isotherms obey the simple power law given by Eq. (9).

To determine the uncertainty in $\rho^{\prime}$ introduced by experimental scatter within a single run, we have analyzed the data as follows: Choosing four isotherms at different temperatures, but done by the same technique for analysis (runs 28, 29/1, 29/2, and 29/3), the data for each was subjected to a simple one parameter least-squares best fit to Eq. (9), with $a(T)$ as the adjustable parameter and $\rho^{\prime}$ fixed. For each value of $\rho^{\prime}, \sigma$ was computed [Eq. (12)]. Plots of $\sigma$ vs $\rho^{\prime}$ for these isotherms may be seen in Fig. 18. The statistical limits on $\rho^{\prime}$ from a single isotherm are given by the values of $\rho^{\prime}$ for which $\sigma$ is equal to

$$
\sigma=\sigma_{0}\left[1+(N-m-1)^{-1}\right]^{1 / 2} \text {, }
$$

where $\sigma_{0}$ is the minimum value of $\sigma$. Here $m=1$

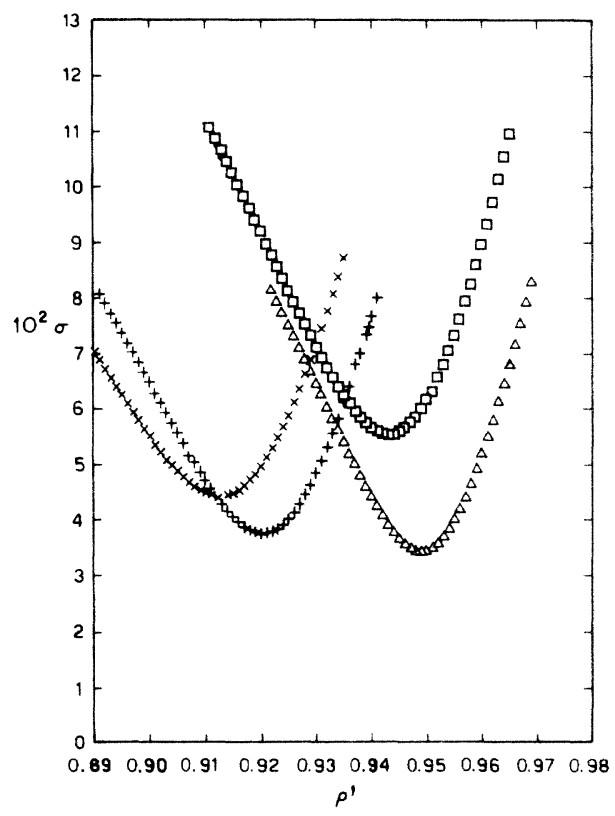

FIG. 18. Plot of the standard error o [Eq. (12)] for a one-parameter least-squares best fit to Eq. (9) as a function of the critical exponent $\rho^{\prime}$ [Eq. (7)] for four different runs: $\times, T=2.006 \mathrm{~K}$ (run 28$) ;+, T=2.094 \mathrm{~K}$ (run 29/1); $\triangle, T=1.904 \mathrm{~K}$ (run 29/2); $\square, T=1.804 \mathrm{~K}$ (run 29/3). and $N \approx 15$ for each isotherm, so $\sigma$ in Eq. (14) is only about $3 \%$ above $\sigma_{0}$. Thus, the statistical uncertainties in $\rho^{\prime}$ in each run-roughly $\pm 0.5 \%$-are small compared to the differences between the best values of $\rho^{\prime}$ in different isotherms.

In Fig. 19 we plot the best values of $\rho^{\prime}$ from these runs as a function of temperature. A point extracted from the saturated vapor pressure data, transformed into an effective isotherm and analyzed in the same way is also included. There is no apparent systematic trend, but we cannot, of course, rule out the possibility that $\rho^{\prime}$ changes with temperature and pressure along the $\lambda$ line. If we suppose, instead, that these are all measurements of the same number, we can take the average value which is $\rho^{\prime}=0.94$. In Fig. 20 we plot the best-fit values of $a(T)$, both for the value of $\rho^{\prime}$ that minimizes $\sigma$ in each isotherm, and for $\rho^{\prime}$ fixed at 0.94 . In the latter case, unlike the former, there is a systematic trend, and we can fit $a(T)$ to $\pm 3 \%$ by

$$
a(T)=27-6.67 T \text {. }
$$

The fact that the single choice $\rho^{\prime}=0.94$ renders $a(T)$ systematic lends more credence to the idea that $\rho^{\prime}$ ought to be taken as constant all along the $\lambda$ line.

We have not analyzed all of the data by this laborious process. Instead, all of the isotherms (including the ones just discussed) were subjected to a two parameter least-squares best fit, with $\rho^{\prime}$ and $\log _{10} a(T)$ as parameters

$$
\log _{10}\left[(\Delta \mu / \mu)_{T}\right\rfloor=\rho^{\prime} \log _{10}(-\epsilon)+\log _{10} a(T) .
$$

The value of $a(T)$ obtained in this way is not of interest, since as we have seen it is sensitive to the choice of $\rho^{\prime}$, but we do obtain for each isotherm a $\rho^{\prime}$ comparable to those discussed above. In doing this analysis it was necessary to take into account the large errors in those points at small $-\epsilon$.

These errors are due both to uncertainties in the

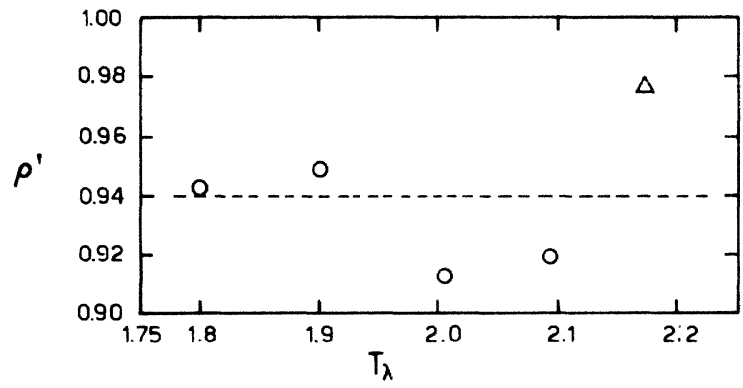

FIG. 19. Plot of the best values of the critical exponent $\rho^{\prime}$ as a function of the temperature $T$. The point at $T=2.172 \mathrm{~K}$ is extracted from the data at saturated vapor pressure, transformed into an effective isotherm [Eq.(13)]. 
identification of the $\lambda$ point parameters in each run, and to fluctuations in the regulated bath temperature. As discussed in Sec. III, the combined effect is to introduce an uncertainty in $-\epsilon$ of about $2 \times 10^{-5}$, and corresponding uncertainty in $\Delta \mu / \mu$. Rather than computing appropriate weighting factors for each data point to be used in the best fit calculations, we simply made use in the calculations only of data with $-\epsilon>1.5 \times 10^{-4}$, where these errors are negligible. For orientation, the effects of these errors are shown by the error bars in Fig. 12, where all of the data points have been plotted.

Values of $\rho^{\prime}$ obtained by best fit to Eq. (16) are plotted in Fig. 21 using different symbols according to the technique used to null the current and correct for drifts, as discussed in Sec. III. There are no apparent correlations between the resulting value of $\rho^{\prime}$ and either the temperature along the $\lambda$ line, or the technique used.

We conclude from these analyses that the drifts and related experimental difficulties introduce into the results errors which are systematic within a single run (giving little statistical error in $\rho^{\prime}$ ), but random from run to run. The most sensible attitude at this point seems to be to suppose that we have made ten independent measurements of the same quantity $\rho^{\prime}$. In this case, averaging the results gives

$$
\rho^{\prime}=0.940 \pm 0.021 \text {. }
$$

The average value thus does not change whether we use five isotherms made with the same technique, or all ten isotherms using various techniques, indicating that there is no systematic bias in $\rho^{\prime}$.

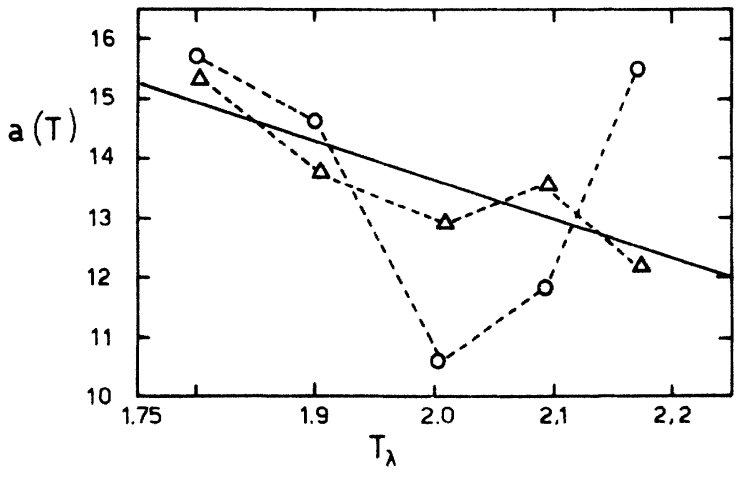

FIG. 20. Plot of the best values of $a(T)$ [Eq. (9)] as a function of the temperature $T$. The open circles represent values' of $a(T)$ using for $\rho^{\prime}$ the value which minimizes $\sigma$ in each isotherm (see Fig. 18); the triangles represent values of $a(T)$ obtained with a fixed average value of $\rho^{\prime}\left(\rho^{\prime}=0.94\right)$.
These conclusions, of course, depend on the assumption that Eq. (9) is obeyed along isotherms. If instead, a pure power law is obeyed along an isochore, or an isobar, or some path close to those, all of the values of $\rho^{\prime}$ will be reduced by 1 or $2 \%$, as will the average value.

It should be mentioned that an attempt was made to see if the data could be fitted by a law of the form

$$
(\Delta \mu / \mu)_{T}=C_{1}(-\epsilon) \log _{10}(-\epsilon)+C_{2}(-\epsilon) .
$$

Such behavior would mean that the divergence in the slope of $\mu$ at the $\lambda$ line would be logarithmic in $-\epsilon$. A two parameter best fit was tried on all the isotherms just like that for the power law summarized in Table I. The resulting values of $\sigma$ were in all cases much higher than those for the best choice of $\rho^{\prime}$ and, in fact, were close to the values of $\sigma$ obtained for $\rho^{\prime}=1$. We thus reject the form given in Eq. (18).

\section{CONCLUSIONS}

We have presented and discussed a new technique for measuring small changes in ion mobilities. This technique renders it possible to make measurements that are sufficiently close to the $\lambda$ transition in helium to be analyzed for critical behavior. On the basis of the results now available, we have been able to conclude that ion mobilities are singular at the transition, in the sense of coming into the $\lambda$ line from the superfluid side with infinite slope. Most of the results are for positive ions, but negative ions appear to behave in the

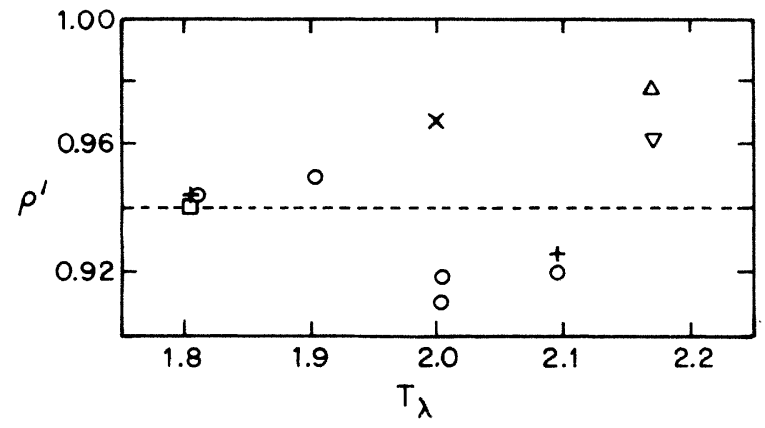

FIG. 21. Values of the critical exponent $f^{\prime}$ obtained by best fit to Eq. (16) plotted against the temperature of the isotherms. Different symbols correspond to different techniques used to null the current (see Sec. III) and corrections for drift, as follows: $\times$, nulling method $B$, not corrected for drift (run 14); $\square$, nulling method $B$, corrected for drift (run 16); + , nulling method $C$, not corrected for drift (runs 23, 25); $\bigcirc$, nulling method $C$, corrected for drift (runs 27, 28, 29/1, $29 / 2$, and 29/3); $\triangle$, data along the vapor pressure curve corrected to isotherm [Eq. (13)]. 
same way.

Experimental errors together with the nature of the singularity itself make quantitative analysis of the singularity somewhat uncertain. However, if we assume a dependence of the type Eq. (9), then we find a critical exponent for ion mobilities whose average value is given by Eq. (17), and with even less certainty, a coefficient given by Eq. (15).

Previous measurements of ion mobilities in the general vicinity of the $\lambda$ transition have usually been interpreted in terms of Stokes law, the ion being thought of as a sphere in a viscous fluid. The mobility of positive ions is then given by

$$
\mu=e(6 \pi \eta r)^{-1} \text {, }
$$

where $e$ is the electron charge, $\eta$ the viscosity, and $r$ the ion radius. Under this assumption, if $r$ is constant, then $\left(\eta-\eta_{\lambda}\right) / \eta_{\lambda}$ ought to have the same limiting behavior as $\Delta \mu / \mu$. Ahlers ${ }^{20}$ has analyzed the existing data for $\eta$ and finds it consistent with

$$
\left(\eta-\eta_{\lambda}\right) / \eta_{\lambda} \sim(-\epsilon)^{0.85}
$$

on the superfluid side of the transition. It is difficult to say whether the difference between this exponent 0.85 and the somewhat higher value we report is significant. Higher resolution measurements of both $\eta$ and $\mu$ will be necessary to resolve the question. Ahlers and Gamota ${ }^{9}$ form the term $(\mu \eta)^{-1}$ which should be proportional to the effective ion radius, and find evidence for singular behavior in that, but the analysis is based on data that fall relatively far from the transition.

A complete experimental determination of the behavior of ions at the phase transition will require at least an order of magnitude improvement in resolution for results of the kind presented here, together with more extensive investigation of negative ions, and most importantly, measurements on the normal side of the transition. Work is in progress along these lines and may be expected eventually to emerge. The importance of the first generation results presented here is first to show that critical measurements of ion mobilities are now possible, and second, that the ions behave in a singular manner at the transition.

Traditionally, ions have been an important source of information about the behavior of liquid helium. More recently, the properties of the $\lambda$ transition have emerged as a key test of general ideas about critical phenomena, at least insofar as equilibrium properties are concerned. The behavior of transport properties at the transition, and theories concerning them, such as the dynamic scaling laws, are much less well established. Ion mobilities in the critical region at the $\lambda$ transition may now be thought of as a measurable transport property. There is every reason to hope that the results will eventually attain the resolution now found only in measurements of such equilibrium properties as the heat capacity and the superfluid fraction. There is, at present, no theoretical work known to us on how ions ought to behave at the transition. We hope the present results will stimulate such investigations.

\section{ACKNOWLEDGMENTS}

We are happy to acknowledge that we have benefitted from the skilled technical assistance of Luciano Mori and from some very enjoyable discussions with Dr. Guenther Ahlers.
*Work supported by CNEN and by NSF Grant No. GH 34682. This cooperative effort has also been facilitated by the appointment of one of us (D. Goodstein) as a Sloan Research Fellow (1970-71) and a NATO Visiting Professor (1972).

$\dagger$ Permanent address.

${ }^{1}$ For a general review, see H. E. Stanley, Introduction to Phase Transitions and Critical Phenomena (Oxford U. P., New York, 1971).

${ }^{2}$ A. Fote, H. Lutz, T. Mihalisin, and J. Crow, Phys. Lett. A 33, 416 (1970)

${ }^{3}$ F. C. Zumsteg and R. D. Parks, Phys. Rev. Lett. 24, 520 (1970); P. P. Craig, W. I. Goldburg, F. A. Kitchens, and J. I. Rudnick, ibid. 19, 1334 (1967).

${ }^{4}$ D. S. Simons and M. B. Salomon, Phys. Rev. Lett. 26, 750 (1971).

${ }^{5}$ A. Stein and F. Allen, Phys. Rev. Lett. 29, 1236 (1972).

${ }^{6} \mathrm{M}$. Fisher and J. S. Langer, Phys. Rev. Lett. 20, 665 (1968).

${ }^{7}$ D. T. Grimsrud and F. Scaramuzzi, Proceedings of the
Tenth International Conference on Low Temperature Physics (Viniti, Moscow, 1967).

${ }^{8}$ D. M. Sitton and F. Moss, Phys. Lett. A 34, 159 (1961).

${ }^{9}$ G. Ahlers and G. Gamota, Phys. Lett. A $\underline{38}, 65$ (1972).

${ }^{10} \mathrm{G}$. Ahlers, Phys. Rev. A $\underline{3}, 696$ (1971).

${ }^{11}$ D. Greywall and G. Ahlers, Phys. Rev. Lett. $\underline{28}, 1251$ (1972).

${ }^{12}$ G. Terui and A. Ikushima, Phys. Lett. A 39, 161 (1972).

${ }^{13}$ Precision pressure gauge, Model No. $145-01$, produced by Texas Instruments.

${ }^{14} \mathrm{~F}$. Scaramuzzi, Internal Report LNF 63/79, CNENRoma (unpublished).

${ }^{15}$ H. F. Ivey, Adv. Electron. Electron Phys. 6, 137 (1954).

${ }^{16}$ Vibrating reed electrometer, "Master and Slave" configuration, Model No. 31, produced by Cary.

${ }^{17}$ H. A. Kierstead, Phys. Rev. 162, 153 (1967).

${ }^{18} \mathrm{H}$. A. Kierstead, Phys. Rev. $\overline{153}, 258$ (1967).

${ }^{19} \mathrm{G}$. Ahlers, The Physics of Liquid and Solid Helium

(Wiley, N.Y., to be published), Chap. VIII.

${ }^{20} \mathrm{G}$. Ahlers, Phys. Lett. A37, 151 (1971). 